\title{
The Forestry Enterprises Emergency Management: Based on Knowledge Element for Emergencies Linking Technology
}

\author{
Shang Hangbiao ${ }^{1, a}$, Tian Guoshuang ${ }^{1, b}$, Qu Wenyu ${ }^{1, c}$, Chen Lirong ${ }^{1, d}$ \\ ${ }^{1}$ Northeast Forestry University, Harbin, 150040, China \\ aemail: shbszz@163.com, bemail:tgs1963@sina.com, 'email:quwenyu@163.com, \\ bemail:freefly1981@163.com
}

Keywords: Emergency Document; Knowledge Element; Emergency Management; Knowledge Linking

\begin{abstract}
This paper builds plans for emergency management of forestry enterprises, and provide a new effective use emergency document for forestry enterprises to respond to emergenciesway based on the knowledge element linking technology, because the emergency management of forestry enterprises can not be quickly and efficiently and find emergency document the problem. Through the document structured analysis, extracted emergency document knowledge element. RDF to integrate the knowledge element analysis, the semantics associated with the completion of the extraction of the relationship between the knowledge element. Finally, the knowledge element linking design plans for emergency management of forestry enterprises. The one hand, the results of this study can guide forestry enterprises emergency practice, on the other hand has laid a foundation for the visual design of the emergency management of forestry enterprises.
\end{abstract}

\section{Introduction}

How to implement Forest industry enterprises for emergencies emergency management has traditionally been party committees, governments, and forestry departments at all levels are the most care and worry the big and hard. In view of the sudden events, Forest industry enterprises how to implement emergency management? But in the face of the sudden event emergency management need to first clear the following questions: (1) what departments and personnel to need to do? Need them to do? (2) what resources need to requisition? There are two ways to solve these problems: one is relating to the event contingency plans, laws and regulations, such as past case data, according to pre-established process and module to carry out emergency response work; Then it is to seek relevant experts of the intellectual support and guidance.

\section{The Forest industry enterprises emergency management knowledge Element link structure analysis}

As shown in figure 1, the reemployment of enterprise emergency management knowledge Element link building pattern mainly divided into three functional modules: emergency document knowledge element extraction module[1], the relationship between the knowledge element extraction module and knowledge Element link module, the resulting based on Forest industry enterprises emergency management system of knowledge, namely Forest industry enterprises emergency plan[2].

(1)Emergency Document Knowledge Element Extraction Module

Emergency document knowledge element extraction module is mainly to emergency and forest industries[3], for example, forest diseases and insect pests, forest fire enclosed landslides, floods, disasters and all kinds of people and other public crisis events relevant contingency plans, laws, regulations, guidelines, emergency case emergency documents and expert experience, knowledge element extraction. Liao open international [4], such as document information is regarded as A feature vector matrix A. 


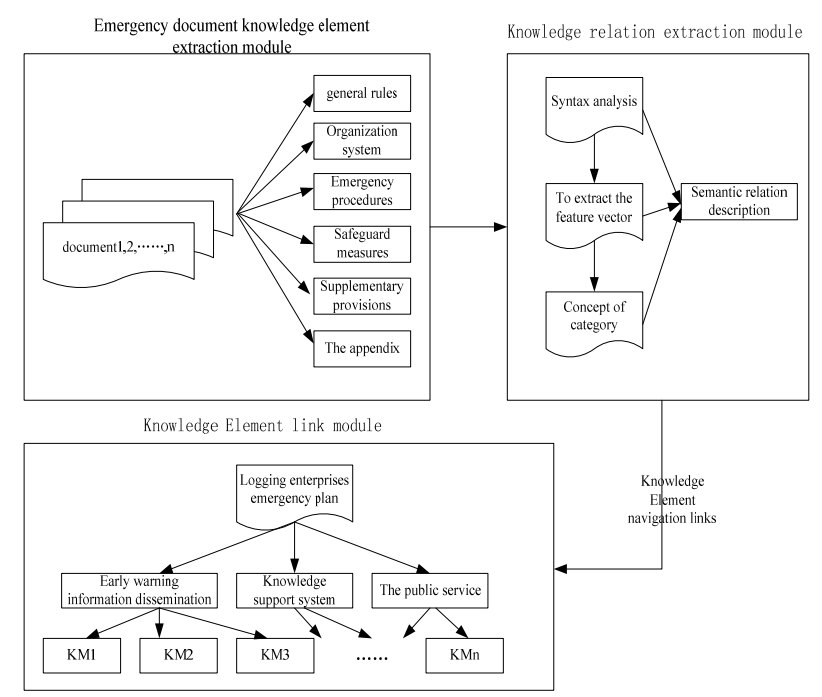

Figure 1. Forest industry enterprises emergency management knowledge link building

$$
A^{\prime}=\left(W_{i j}\right)_{m \times n}=\left[\begin{array}{cccc}
W_{11} & W_{12} & \cdots & W_{1 n} \\
W_{21} & W_{22} & \cdots & W_{2 n} \\
\vdots & \vdots & & \vdots \\
W_{m 1} & W_{m 2} & \cdots & W_{m n}
\end{array}\right]
$$

The row vector is the document vector and column is to retrieve a vector[5]. Refers to the document I weight on retrieval item $\mathrm{j}$. Set up document metadata table storage document information matrix, document metadata table included in the document number, document title, author, and the release date, document properties, events classification, event classification and description of the document content and so on.

(2)Emergency Document Knowledge Relation Extraction Module

Emergency document knowledge relation extraction module mainly is the use of RDF (resource description framework (RDF) technology to integrate knowledge element analysis, establish semantic associations, and relations between the knowledge element extraction [6]. RDF is a kind of on the forms of framework to describe information resources, the knowledge element of the relationship between attributes and attribute are identified, then the method to define knowledge Element a technology.

RDF syntax structure is a three tuple, which it USES the form of triple structure of knowledge element semantic description, and relations between the knowledge element extraction [7]. Ternary group representation: $K_{m}=\left\{N_{m}, A_{m}, R_{m}\right\} \forall m \subset M$, that is to say a knowledge including concept name $\left(N_{m}\right)$, Element structure with different attributes $\left(A_{m}\right)$ and intellectual connection relationship between Element Element with the knowledge attribute ( $R_{m}$ ). Triple form is in the knowledge level of abstract representations of the objective things, realize the semantic description of knowledge Element, achieve knowledge description of semantic relationship between Element, then complete the emergency document knowledge relation extraction.

Specifically, RDF description of knowledge element includes three steps: using RDF syntax analysis was carried out on the document knowledge Element, on the basis of using the string matching optimization algorithm to automatic segmentation of knowledge element, knowledge element to define the concept of a name. the feature vector is extracted, using vector space model and support vector calculation method of feature vector is extracted and stored. According to the research of the manifold to open international etc. [5], Element according to the characteristics can be divided into solving the theme knowledge "about what" question Element event type knowledge, solve the problem of "about the who" type subject knowledge, and resolve "about the who", "about 
when", about how "problem of task-oriented knowledge Element. Event type knowledge feature vector extracted, subject characteristics can be abstracted as" $N I+V L+$ what ", which represents the term entity, said is a verb "is", said the "what"; the subject knowledge feature vector extracted, subject characteristics can be abstracted as " $s u b+V L+w h o$ ", which represents the emergency main body, said is a verb" is ", said the "who"; task-based knowledge feature vector extracted, subject characteristics can be abstracted as " $s u b+$ when $+V+N E M$ ", which represents the emergency, said" when ", said is a "what to do with" action, it is said to deal with emergency problems. using the clustering data mining technology to concept classification feature vector.

(3)Knowledge Element Link Module

Emergency document knowledge relation extraction after the formation of knowledge element semantic description, Element link module through knowledge, based on knowledge element concentration, classification and combination navigation links such as process, generate Forest industry enterprises emergency management subject knowledge system [8]. Knowledge system, refers to the organized by themes emergency management is a set of basic knowledge structure. Knowledge is Element constitute the basic unit of the knowledge structure, they eventually RMB through knowledge navigation links link together [5]. Element knowledge navigation links in essence is a condition on a specific emergency management subjects, based on the correlation between knowledge Element, Element link to build specific knowledge, provide intellectual support for dealing with emergencies [9]. Reasoning process of knowledge Element network link, as shown in figure 2.

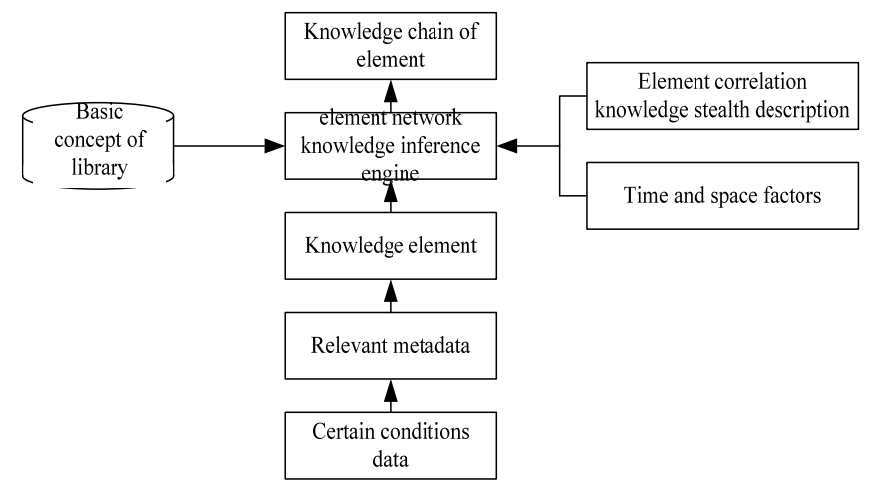

Figure 2. Element network links the reasoning process of knowledge

Specifically, Forest industry enterprises should plan consists of three subsystems, respectively for the early warning information transmission subsystem, knowledge support subsystem and the public service subsystem. Early warning information dissemination subsystems including forest fires, pests, debris flow, flood disasters and other emergencies of warning and defense and so on knowledge and information; Knowledge support subsystem is refers to the management of emergency department staff need to understand and retrieve when faced with emergency document meta information, knowledge and expert information, etc.; Public service subsystem is geared to the needs of the social public, supplying them with emergency knowledge. Forest industry enterprises emergency preplan knowledge Element link diagram, as shown in figure 3.

\section{Conclusion}

Based on the theory of knowledge Element link, according to the characteristics of Forest industry enterprises emergency management, in the collection and research of Forest industry enterprises emergency document features, based on the study of the knowledge of Forest industry enterprises emergency link model. Specifically, through the document structured analysis, extract knowledge Element emergency documents. Using RDF to integrate knowledge element analysis, the establishment of semantic association, complete the knowledge relation extraction. Finally, knowledge link, reemployment of enterprise emergency management plan is designed. Forest industry enterprises emergency management knowledge Element link building pattern mainly 
divided into three functional modules: emergency document knowledge element extraction module, the relationship between the knowledge element extraction module and knowledge Element link module. Based on the three modules, design the reemployment of enterprise emergency management plans, including early warning information transmission subsystem, knowledge support subsystem and the public service subsystem.

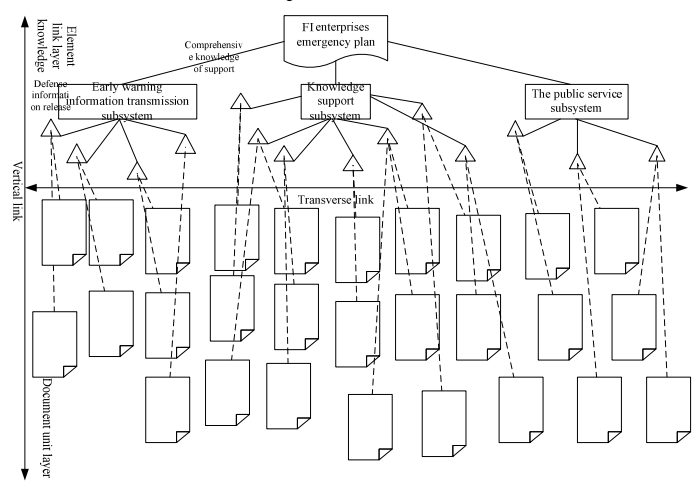

Figure 3. Forest industry enterprises emergency preplan Element link schematic knowledge

\section{Acknowledgment}

This paper was funded by projects for Humanities and Social Sciences of Department of Education of Heilongjiang Province(Project No. 12514026), and the Fundamental Research Funds for the Central Universities (Project No. DL11CC05).

\section{References}

[1] Jiang Yongchang., Yang Hongyan., Zhang Libo. Based on knowledge element of knowledge organization and system service function research [J]. Journal of theory and exploration, 2007, 30 (1) : 37-40

[2] Wen Youkui., Xu Guohua. Knowledge element link theory [J]. Journal of intelligence, 2003, (12) : 665-670.

[3] Teng GuangQing., Bi Jiang. The inner mechanism of the knowledge link and learn source evolution research [J]. Journal of theory and practice of intelligence, 2010 (2) : 21-24.

[4] Zhong QiuYan.,Guo Yanmin., Wang Ning., Xue Huifang., Cui Li., Wang Yanzhang. The unconventional emergency scene model based on knowledge element research [J]. Journal of intelligence science, 2012, 30 (1) : 115-120.

[5] liao Kaiji., Xiong Huihui., Ye Donghai. Emergency document structured modeling based on knowledge element theory [J]. Computer application research, 2011, 28 (1) : 175-178.

[6] Wen kui., JiaoYuYing. Knowledge semantic link model research [J]. Journal of library intelligence, 2010, (12) : 27-31.

[7] XiaoHong., Xue Dejun. Based on the large scale real texts numerical knowledge element mining research [J]. Computer engineering and applications, 2008, 44 (30) : 150-152.

[8] Xie Cuixiang., Zhang Zhonglin., Chen Zhi. A bibliographic query system based on vector space model design [J]. Journal of theory and practice of intelligence, 2007, 30 (2) : 241-243.

[9] Chen Xuelong., Dong Enchao., Wang Yanzhang., XiaoWenHui., Gong jiaqi. Knowledge of unconventional emergency management model [J]. Journal of intelligence magazine, 2011, 30 (12) : 22-26. 